\title{
Dextrose boluses versus burette dextrose infusions in prevention of hypoglycemia among preterms admitted at Mulago Hospital: an open label randomized clinical trial.
}

\author{
Kutamba $E^{1}$, Lubega $\mathrm{S}^{1,2}$, Mugalu $\mathrm{J}^{1,2}$, Ouma $\mathrm{J}^{3}$, Mupere $\mathrm{E}^{1}$
}

1. Department of Paediatrics and Child Health, Makerere College of Health Sciences, Kampala, Uganda,

2. Ministry of Health Kampala Uganda, ${ }^{3}$ Management Sciences for Health-Kampala

\begin{abstract}
Background: Hypoglycemia is a major cause of morbidity and mortality among preterm infants and its management remains a challenge in resource limited settings. Use of dextrose infusion by the recommended infusion pumps is not feasible in our environment due to their high costs and yet the current use of mini dextrose boluses with syringes as adapted at Mulago national referral and tertiary teaching hospital has unknown efficacy in prevention of hypoglycemia

Objective: We determined the efficacy of dextrose infusions by burettes versus two hourly dextrose boluses in prevention of hypoglycemia among preterms admitted in the first 72 hours at Special Care Unit, Mulago Hospital.

Methods: One hundred and forty preterms aged 0 to 24 hours of life were randomized to receive $10 \%$ IV dextrose either as mini boluses or by infusion using burettes in an open label clinical trial. Blood glucose was measured at 0 , two hourly for next 6 hours, 6 hourly for next 12 hours and thereafter 12 hourly until end of 72 hours following admission. Primary end point was incidence of hypoglycemia (random blood sugar (RBS) $<2.6 \mathrm{mmol} / \mathrm{l}$ ) which was expressed as relative risk (RR). Efficacy of the dextrose infusion was computed using 1-RR.

Results: From February 2012 to April 2012, 68 preterms in the bolus arm and 72 in the infusion arm were studied. Hypoglycemia was detected in 34\% (48/140). The incidence of hypoglycemia in the bolus arm was 59\% (40/68) compared to $11 \%(8 / 72)$ in the infusion arm (RR; 0.19, 95\% CI; 0.09-0.37). Efficacy (1-RR) of infusion by burettes versus boluses in prevention of hypoglycemia among preterms was 0.81 (95\% CI; 0.63-0.90).

Conclusion: Continuous 10\% dextrose infusion by burettes reduced the incidence of hypoglycemia by $81 \%$ in the first 72 hours of admission compared to two hourly 10\% mini dextrose boluses among preterms admitted at Special Care Unit, Mulago Hospital.(ClinicalTrials.gov Identifier: NCT01688674)
\end{abstract}

Keywords: Dextrose boluses, burette infusions, hypoglycemia.

DOI:http://dx.doi.org/10.4314/ahs.v14i3.2

\section{Introduction}

Prematurity and low birth weight contribute $29 \%$ of the total newborn deaths globally ${ }^{(1)}$ and hypoglycemia is one of the major complications of prematurity. The overall incidence has been reported to be 1 to 5 per 1000 live births and the risk is highest in preterm infants ${ }^{(2)}$. There is paucity of data regarding incidence of hypoglycemia among preterm infants in developing

\section{Corresponding author:}

Kutamba Elizabeth

Department of Paediatrics and

Child Health,

Makerere College of Health Sciences,

Kampala, Uganda

Email: kutambae@yahoo.com

Mobile +256772640821 countries. Prematurity accounts for $25 \%$ of newborn deaths in Uganda ${ }^{(3)}$. Challenges in prevention of hypoglycemia may contribute to the high mortality among preterm infants. The World Health Organization (WHO) recommends early initiation of breastfeeding (first one hour) as the most effective method of preventing hypoglycemia in newborns. However where enteral feeding is not possible, the WHO recommends continuous $10 \%$ dextrose by infusion pumps to prevent hypoglycemia among newborns ${ }^{(4)}$. Most resource limited settings may not afford infusion pumps to administer continuous 10\% dextrose. Currently dextrose boluses of $10 \%$ given every two hours using disposable syringes referred to as mini dextrose boluses have been adapted at the national referral and tertiary teaching Special Care Unit Mulago hospital, Kampala, Uganda ${ }^{(5)}$. However efficacy of mini dextrose at preventing hypoglycemia in Uganda is unknown to justify the continued use. Burette infusion sets which are cheaper and readily 
available in developing countries may be an alternative in administering continuous $10 \%$ dextrose.

We undertook an open label randomized clinical trial to assess whether IV 10\% dextrose given as mini boluses compared to burette infusion had similar efficacy in prevention of hypoglycemia among preterm infants.

\section{Methods:}

\section{Study design and setting.}

This was an open label randomized clinical trial. The study was conducted at Mulago Hospital's special care unit, Uganda's teaching and referral hospital. It admits both full term and preterm infants for specialized care from the capital city and referrals from other parts of the country.

\section{Outcome measures}

The primary outcome measure was incidence of hypoglycemia to establish the efficacy of administering $10 \%$ dextrose using two hourly mini boluses versus continuous infusion by burettes in prevention of hypoglycemia. A preterm was considered to have developed hypoglycemia if the random blood sugar (RBS) was $<2.6 \mathrm{mmol} / \mathrm{l}$. The measure for efficacy was incidence proportion, which was determined by the proportion of new cases of hypoglycemia. This was expressed as a relative risk (RR) and efficacy was calculated as 1-RR.

The secondary outcome was observed survival of preterms at the end of the 72 hour period.

This was measured as the proportion of patients remaining alive from the time of enrolment until end of 72 hours.

\section{Ethical issues}

Permission to conduct the study was obtained the Makerere School of Medicine Research and Ethics Committee (SOMREC). Written consent was obtained from the mothers for enrollment into the study. A data and safety monitoring board (DSMB) was set up to oversee to activities of the research.

\section{Sample size}

We planned an interventional study of preterm infants receiving dextrose by either burette infusion sets or boluses that are routinely used in the neonatal unit at Mulago hospital. Prior data indicated that the incidence of hypoglycemia in the infusion arm was $27 \%{ }^{(6)}$, If the boluses increased the incidence of hypoglycemia by $30 \%$ and true relative risk of hypoglycemia in the infusion arm was 0.77 relative to the bolus arm, we needed to study 84 patients in infusion arm and 84 patients in the bolus arm to be able to reject the null hypothesis that the relative risk equals 1 with probability (power) of 0.9. The type I error probability associated with the test of this null hypothesis was assumed to be 0.05 . Thus, a total of 168 preterm infants were to be enrolled into the study. We used an uncorrected chi-squared statistic to evaluate the null hypothesis. Sample size calculation was performed using PS (Power and size) software ${ }^{(7)}$

This sample size was adequate to carry out a three stage interim analysis. The first analysis was done at 50\% accrual; the second analysis was done at $75 \%$ and the final analysis at the end of the study.

\section{Participants and recruitment}

We enrolled preterms aged 0 to 24 hours of life admitted to the Mulago's special care unit from February to April 2012 with a RBS of $\geq 2.6 \mathrm{mmol} / 1$ and birth weight of $\geq 1 \mathrm{~kg}$. We excluded all preterms who had gross congenital abnormalities, very sick (Apgar score $\leq 5$ at 5 minutes) and an abandoned or orphaned preterm infant at the time of recruitment.

Trained research nurses consented patients, monitored random blood sugar and administered the interventions. The principal investigator also participated in recruitment, and monitoring of study participants and ensuring study protocols were strictly followed. Blood sugar was tested using a digital glucometer (Optium Xceed, Abbott Laboratories Ltd).

\section{Randomization, blinding and treatment allocation}

Eligible preterm infants were enrolled in a serial order and randomized to receive $10 \%$ dextrose either by burette infusions or two hourly mini boluses for 72 hours during admission into the Mulago Hospital's Special care unit. The randomization list was generated using a computer by a statistician not involved in the implementation of the study. Block randomization was used with a block size of four. Randomization codes indicating the treatment arm were sealed in opaque envelopes which were sequentially numbered. The study nurse gave the intervention according to the envelopes.

\section{Intervention}

The use of 2 hourly 10\% dextrose mini boluses was the standard of care in Mulago hospital and the infusion by burette sets was the experimental arm under study. Intravenous $10 \%$ dextrose was prepared by adding 50 mls of $50 \%$ dextrose to $450 \mathrm{mls}$ of $5 \%$ dextrose to obtain $500 \mathrm{mls}$ of $10 \%$ dextrose. 
Once an envelope was opened to see the study group to be assigned, the patient had an intravenous cannula inserted and a study nurse administered 10\% dextrose according to protocol.

Daily Fluid requirements of $10 \%$ dextrose were calculated for the 72 hours of follow as $80 \mathrm{mls} / \mathrm{kg} /$ day, $100 \mathrm{mls} / \mathrm{kg} /$ day and $120 \mathrm{mls} / \mathrm{kg} /$ day for day 1,2 and 3 respectively as per SCU protocol (see appendix 5). The infusion rate was calculated as drops per minute and cross-checked every thirty minutes to ensure regular flow. The burette administers 60drops/ml (Poly Vol Burette set manufactured by Poly Medicure Ltd). The infusion rate was calculated every 24 hours for the 3 days of follow up as; [[Total fluid volume $(\mathrm{mls}) \times$ Birth weight $(\mathrm{kg})] / 1440$ minutes] $\times 60=$ drops $/ \mathrm{min}]$. For the preterms in the bolus arm, the total volume was given as two hourly boluses each pushed over 1 minute (total volume required per $\mathrm{kg}$ body weight per 24 hours divided by 12 ; to obtain the volume of $10 \% \mathrm{D}$ to be given every two hours).

\section{Blood glucose monitoring}

Blood sugar tests were done at time of recruitment, every 2 hourly for the first 6 hours then 6 hourly until end of the first 12 hours and then 12 hourly until 72 hours of admission. For preterms in the bolus arm blood sugar testing was done before administration of dextrose boluses. At the end of the study period the preterms were handed over to the clinical team in SCU to continue with routine care. After the initial episode of hypoglycemia, the hypoglycemia was treated by giving them IV 10\% dextrose boluses and the preterms were still followed until the end of 72 hours of admission to determine survival. Preterms who were found to have hyperglycemia (blood sugar $>10 \mathrm{mmol} / \mathrm{l}$ ) had their blood glucose tested every 30minutes until the blood glucose was below $10 \mathrm{mmol} / \mathrm{l}$.

\section{Follow up}

Preterm infants were followed up until discharge, death or a maximum of 72 hours

\section{Data management and analysis:}

Data was entered on Case Report Forms (CRFs), cleaned and entered into the computer using Microsoft Access 2007. The Data was then exported to STATA version 10 for analysis. Analysis was by intention-to-treat method. Data were summarized in form of proportions, percentages or frequencies and presented in frequency tables. Incidence proportion of hypoglycemia with the associated $95 \%$ CIs was determined for the two study arms. Incidence proportion and relative risk were determined using chi-square tests. The efficacy of $10 \%$ dextrose infusion by burettes compared to two hourly $10 \%$ dextrose boluses was calculated using the following expression: 1- RR. ( $R R=$ Relative Risk)

Sub-analysis was done for overall hypoglycemic distributions for infants receiving boluses or infusions. These were estimated using the Kaplan-Meier method and compared using the log-rank test. The time to development of hypoglycemia and the intervention status were used as the primary dependent and independent variables, respectively in the model. The secondary objective was the proportion of preterms surviving by the end of 72 hour period of follow up. Interim analysis done at 75\% recruitment was reviewed by the DSMB and recommended to stop the trial because of overwhelming benefit by the dextrose infusions as compared to the two hourly dextrose boluses.

\section{Results:}

Between February and April 2012, 260 preterms aged less than 24 hours of life were screened for eligibility and of these, 115 (44\%) had hypoglycemia at admission and 5 were excluded because they were very sick. Of the $140(54 \%)$ preterms who were enrolled into the study 68 were randomized into the mini bolus arm, and 72 into the infusion arm and analyzed respectively. The mean gestational age (weeks) was $31.4(\mathrm{SD}=2.7)$ in bolus arm and $31.2(\mathrm{SD}=2.2),(\mathrm{p}=0.655)$ in infusion arm, and mean RBS at enrolment $(\mathrm{mmol} / \mathrm{l})$ was $3.9(\mathrm{SD}=1.3)$ in bolus arm and $4.1(\mathrm{SD}=1.5),(\mathrm{p}=0.723)$ in infusion arm. The mean age (hrs) was 2.3(SD=2.5) and $2.6(\mathrm{SD}=2.8)$, $(p=0.512)$ in bolus arm and infusion arm respectively. The mean birth weight $(\mathrm{Kg})$ was $1.8(\mathrm{SD}=0.4)$ in bolus arm and $1.7(\mathrm{SD}=0.3),(\mathrm{p}=0.137)$ in infusion arm. Mean temperature $\left({ }^{0} \mathrm{C}\right)$ was $34.4(\mathrm{SD}=4.3),(\mathrm{p}=0.989)$ in bolus arm and $34.4(\mathrm{SD}=4.4)$ in infusion arm and the mean Apgar score was $8.0(\mathrm{SD}=1.2)$ in bolus arm and 8.2 $(\mathrm{SD}=1.2),(\mathrm{p}=0.534)$ in infusion arm. There were more males $(52.9 \%)$ than females $(47.1 \%)$. Except for more preterms in the bolus arm who initiated enteral feeding $<48$ hrs than those in the infusion arm there was no statistical difference in the baseline characteristics (Table 1A\&B). 
Table 1 A: Neonatal baseline characteristics among preterms receiving $10 \%$ dextrose in the $1^{\text {st }} 72 \mathrm{hrs}$ of admission at SCU-Mulago Hospital, 2012

\begin{tabular}{|c|c|c|c|}
\hline \multirow[t]{2}{*}{ Variable } & \multirow{2}{*}{$\begin{array}{l}\text { Bolus } \\
n=68(\%)\end{array}$} & Infusion & \multirow[t]{2}{*}{ P-value } \\
\hline & & $\mathrm{n}=72 \quad(\%)$ & \\
\hline \multicolumn{4}{|l|}{ Sex of the baby } \\
\hline Male & $36(48.7)$ & $38(51.4)$ & \\
\hline Female & $32(48.5)$ & $34(51.5)$ & 0.985 \\
\hline \multicolumn{4}{|l|}{ Birth type } \\
\hline Singleton & $49(48.5)$ & $52(51.5)$ & \\
\hline Twin + & $19(52.8)$ & $17(47.3)$ & 0.660 \\
\hline \multicolumn{4}{|l|}{ Size at birth } \\
\hline $\begin{array}{l}\text { SGA } \\
\text { LGA }\end{array}$ & $\begin{array}{l}5(41.8) \\
16(51.6)\end{array}$ & $\begin{array}{l}7.0(58.3) \\
15(48.4)\end{array}$ & \\
\hline AGA & $47(48.5)$ & $50(51.6)$ & 0.842 \\
\hline \multicolumn{4}{|l|}{ Sucking reflex } \\
\hline $\begin{array}{l}\text { Poor } \\
\text { Good }\end{array}$ & $\begin{array}{l}21(44.7) \\
47(50.5)\end{array}$ & $\begin{array}{l}26(55.3) \\
46(49.5)\end{array}$ & 0513 \\
\hline Mode of delivery & & & \\
\hline SVD & $48(50.5)$ & $47(49.5)$ & \\
\hline $\begin{array}{l}\mathrm{C} / \mathrm{S} \\
\text { Start of Enteral feeding }\end{array}$ & $15(39.5)$ & $23(60.5)$ & 0.249 \\
\hline$\leq 48 \mathrm{hrs}$ & $50(83.3)$ & $45(67.2)$ & \\
\hline$>48 \mathrm{hrs}$ & $10(16.7)$ & $22(32.8)$ & 0.036 \\
\hline
\end{tabular}

Table 1B: Maternal baseline characteristics among preterms receiving $10 \%$ dextrose in the $1^{\text {st }} 72 \mathrm{hrs}$ of admission at SCU-Mulago Hospital, 2012.

\begin{tabular}{lccc}
\hline Variable & Bolus (\%) & Infusion (\%) & P-value \\
\hline Marital status & & & \\
Married & $53(50.0)$ & $53(50.0)$ & \\
Not married & $13(43.3)$ & $17(56.7)$ & 0.519 \\
Attended ANC & & & \\
Yes & $63(49.2)$ & $65(50.8)$ & 0.574 \\
No & $4(40.0)$ & $6(60.0)$ & \\
HIV status & $9(47.4)$ & $10(52.6)$ & \\
Positive & $50(48.1)$ & $54(51.9)$ & 0.987 \\
Negative & $8(50.0)$ & $8(50.0)$ & \\
Mothown & & & \\
Drug use & $5(41.8)$ & $7(58.3)$ & \\
Yes & $62(49.2)$ & $64(50.8)$ & 0.618 \\
No & & & \\
Intrapartum Dextrose use & $24(52.2)$ & $22(47.8)$ & \\
Yes & $44(47.3)$ & $50(53.2)$ & 0.551 \\
No & & & \\
Parity & $26(50.9)$ & $25(49.0)$ & \\
Primegravida & $47(52.8)$ & 0.660 \\
Multigravida & & & \\
Residence & Rural & $14(53.9)$ & \\
Urban & $12(46.1)$ & $58(52.3)$ & 0.884 \\
\hline
\end{tabular}

The total time of follow up to development of hypoglycemia among study participants was 8112 person hours $(3320$ and 4792 person hours among the bolus and infusion arms respectively). Forty eight (48) out of one hundred and forty (140) patients developed hypoglycemia with the overall incidence of $34 \%$. Forty 
out of sixty eight $(59 \%)$ preterms in the bolus arm, compared to eight out of seventy two (11\%) in the infusion arm developed hypoglycemia.
Time to development of hypoglycemia was shorter among the preterms receiving 10\% dextrose mini boluses as compared to those in the infusion arm (Figure 2).

Figure 2. Time to development of hypoglycemia among preterms receiving $10 \%$ dextrose by either two hourly boluses or continuous infusion by burettes

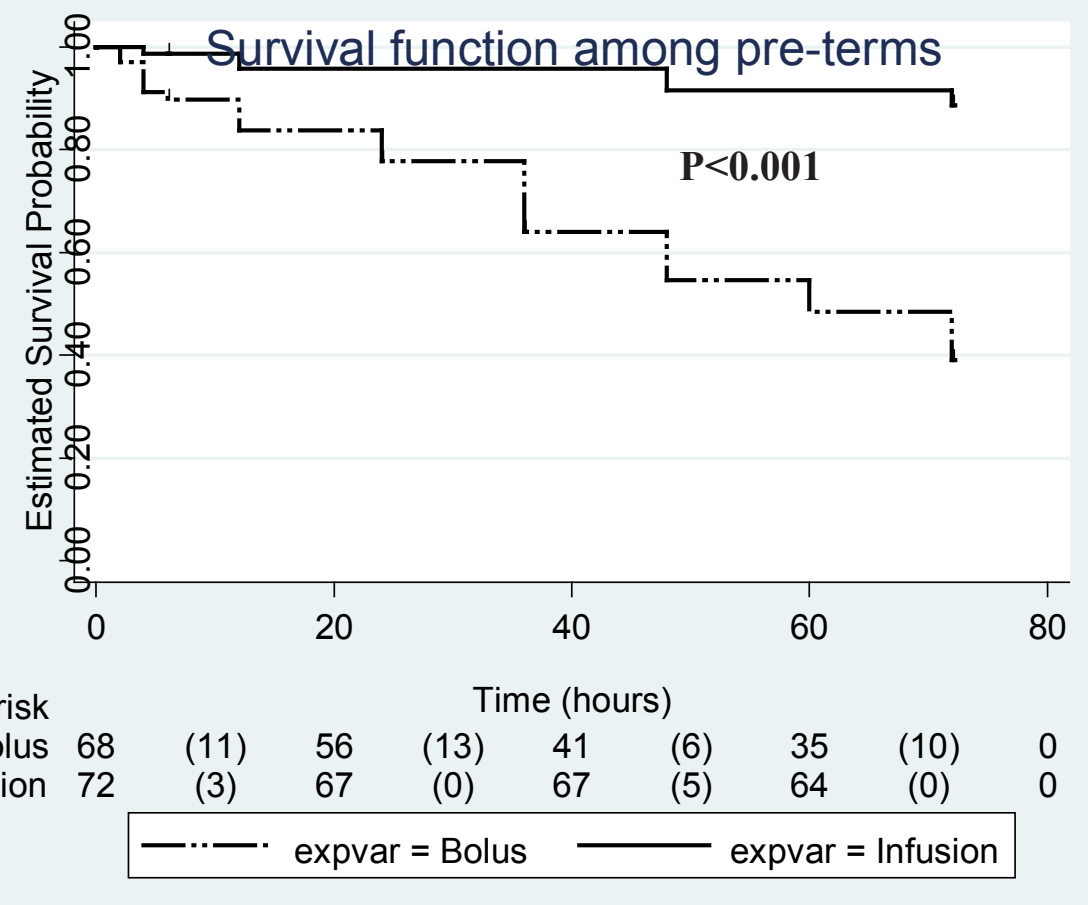

When compared with the $10 \%$ dextrose boluses, $10 \%$ dextrose infusion by burettes reduced the incidence of hypoglycemia by $81 \%$ among preterms admitted in SCU in the first 72 hours (Table 2).

Table 2: Incidence proportion of hypoglycemia among preterms admitted in the 1 st $72 \mathrm{hrs}$ of admission at SCU-Mulago Hospital, 2012

\begin{tabular}{llll}
\hline & Infusion $(\mathrm{n}=72)$ & Bolus $(\mathrm{n}=68)$ & $(95 \% \mathrm{CI})$ \\
\hline Hypoglycemia & 8 & 40 & \\
Incidence proportion & 0.11 & 0.59 & $0.09-0.37$ \\
Relative Risk (RR) & 0.19 & & $0.63-0.90$ \\
Efficacy (1-RR) & 0.81 & & \\
\hline
\end{tabular}

The overall survival among preterms was high at $97 \%(136 / 140) .70 / 72(97.2 \%)$ in the infusion arm compared to $66 / 68(97.1 \%)$ preterms in the bolus arm that survived. Only four deaths (4/140) occurred during the study period and none had hypoglycemia. Among the preterms who died, three out of four $(75 \%)$ had hyperglycemia (RBS $>10 \mathrm{mmol} / \mathrm{l})$. The mean age $( \pm \mathrm{SD})$ among preterms who died was 1.8 (1.5) hours compared to 2.5 (2.7) hours among those who survived 
Figure 1. Trial profile

260 preterms were screened

for eligibility

120 excluded

- $\quad 115-\mathrm{RBS}<2.6 \mathrm{mmol} / \mathrm{1}$

- $4-\mathrm{A} / \mathrm{S} \leq 5$ at $5 \mathrm{mins}$.

$\underset{\downarrow}{140 \text { preterms }} \longrightarrow \cdot 1$ - gross abnormalities

were enrolled and

randomized

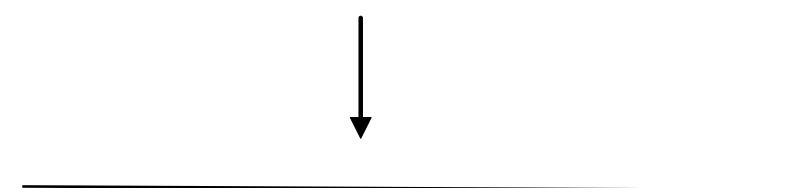

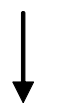

68 preterms received D10 by Boluses

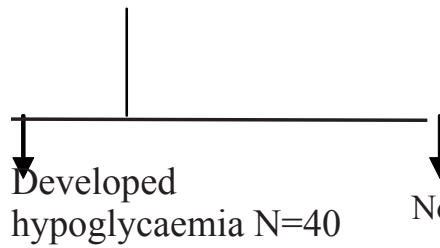

$\mathrm{N}=28 *$
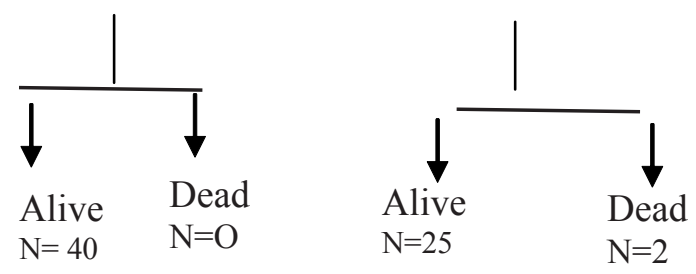

72 preterms received D10 by burette infusion sets

*1 preterm was loss to follow up (Bolus arm)

\section{Discussion:}

This study was carried out to establish the efficacy of using $10 \%$ dextrose mini boluses versus dextrose infusions by burettes in prevention of hypoglycemia among preterms admitted in the first 72 hours at Mulago hospital. The measure of efficacy was the incidence proportion of hypoglycemia in the two study groups, expressed as a relative risk. In this randomized clinical trial, we found that using $10 \%$ dextrose infusion by burettes reduced the incidence of hypoglycemia among preterms by $81 \%$ compared to dextrose boluses.

This implies that $10 \%$ dextrose infusion by burettes is preferred to $10 \%$ dextrose mini boluses in prevention of hypoglycemia of hypoglycemia among preterms. This study is probably the first randomized clinical trial to be conducted in Africa to determine the efficacy of using 10\% dextrose infusion by burettes compared to dextrose boluses in prevention of hypoglycemia among preterms.

The only available study was conducted by Lilien et 
al in USA, who found that 18 out of 22 neonates' glucose levels rose above the hypoglycemic range within ten minutes of initiating 10\% dextrose infusion. He also showed that, constant glucose infusion was therapeutically useful for treatment of neonatal hypoglycemia ${ }^{(8)}$. There are no documented comparative studies that have analyzed the efficacy of $10 \%$ dextrose boluses and 10\% dextrose infusion by burettes in prevention of hypoglycemia among preterms. In this study the overall incidence of hypoglycemia among preterms was high (34\%). Similar to this study, Alaroker et al in Mulago hospital showed a high prevalence (36\%) of hypoglycemia among preterms ${ }^{(9)}$.

The higher incidence of hypoglycemia among preterms in the bolus arm (59\%) could be due to the fact that, when dextrose boluses are administered, the rate of entry into blood exceeds the capacity of body cells to utilize glucose. The excess glucose stimulates the pancreatic $\beta$ cells to secrete insulin and with each succeeding bolus, it decreases the threshold for insulin release but also inhibit glucagon release, thus resulting into hyperinsulinism which causes rebound hypoglycemia ${ }^{(10)}$. It has been recommended by Lilien et al that when dextrose boluses are given to treat hypoglycemia, they should be immediately followed by a continuous dextrose infusion to prevent hyperglycemia with resultant rebound hypoglycemia ${ }^{(11)}$.

The overall survival was high (97\%) in this study and it was similar for both study groups.

On the other hand, Namukwaya et al in a prospective study at Mulago Hospital, showed that hypoglycemia was associated with less survival among very low birth weight infants in SCU $(31.6 \%$ versus $68.3 \%$ in the nonhypoglycemic group), although the association was not statistically significant $(\mathrm{P}=0.498)^{(12)}$. The high survival in this study could be explained by the fact that, this being an interventional study, preterms were actively followed up and treated as soon as hypoglycemia was detected.

It was interesting to note that mortality was high (75\%) among preterms who developed hyperglycemia in this study. This is higher than reported by Vander Lugt Margreth et al in Netherlands who found mortality among the very low birth weight infants with hyperglycemia to be $41 \%{ }^{(13)}$. This study was however not powered to explore factors (including hyperglycemia) associated with mortality.

\section{Adverse events}

Hyperglycemia was regarded as an adverse event in this study. There were almost equal numbers $(29 \%$ in the bolus arm versus $30 \%$ in the infusion arm) of preterms who developed hyperglycemia in both study groups. Most of the preterms who developed hyperglycemia in the infusion arm were found to have a faster infusion rate (drops/min) than previously set.

This is comparable to Louik Carol et al in Boston who described hyperglycemia to occur among infants receiving 10\% dextrose infusion and its risk increased with increasing dextrose dose as well as decreasing birth weight and gestational age $\mathrm{e}^{(14)}$. This study was not powered to explore hyperglycemia and risk factors among preterms.

\section{Study limitations}

This was an open label randomized clinical trial. The measurement of outcome was not blinded to the study nurses and the investigator. However, blood glucose testing was done using a digital glucometer (Optium Xceed, Abbott Laboratories Ltd). It was difficult to interfere with measurement of outcome because the digital glucometer had a memory function that could store the blood glucose value including the time and date of measurement.

Insulin and glucagon blood levels were not measured in this study. These hormones greatly influence glucose metabolism. However, the effect on the results was equally distributed because of randomization.

Although the laboratory determination (hexokinase) of blood glucose is the gold standard for measuring glucose concentration, the glucometer (glucose oxidase) method equally has a high sensitivity (98\%) and specificity (93\%) with positive and negative predictive values of $88 \%$ and $99 \%$ respectively ${ }^{(15)}$. However, the glucometer method may be affected by factors like hemolysis which may be caused by squeezing of the heel to obtain capillary blood.

To minimize this error, squeezing of the heel was avoided and warming of the heel by gentle massage was ensured during collection of capillary blood. However this inherent limitation was distributed in both study groups and therefore had limited influence on the results of this study.

\section{Conclusions:}

Continuous $10 \%$ dextrose infusion by burettes reduced the incidence of hypoglycemia by $81 \%$ compared to two hourly $10 \%$ dextrose boluses among preterms admitted in the first 72 hours in SCU- Mulago Hospital. Survival was high among preterms admitted in the first 72 hours in SCU-Mulago Hospital. 


\section{Recommendations:}

We recommended that Continuous 10\% dextrose infusion by burettes should be considered as an option in prevention of hypoglycemia among preterms admitted in SCU at Mulago Hospital. A study is also recommended to determine the incidence of hyperglycemia and risk factors for mortality among preterms receiving 10\% dextrose.

\section{Acknowledgements:}

We thank all the preterm infants, their parents/care takers and the study nurses who participated in the study.

\section{Conflict of interest:}

None

\section{Authors' contributions:}

Kutamba Elizabeth, Mugalu Jamiir,Lubega Sulaiman and MupereEzekiel participatedin the conception, designand implementation of the study. Ouma Joseph and Mupere Ezekiel were involved in the statistical analysis; Mupere Ezekiel, Lubega Sulaiman, Mugalu Jamiir and Kutamba Elizabeth were involved in drafting the manuscript.

\section{References}

1. World Health Organization (WHO), Major causes of death in newborns and children. 2010.

2. McGowan JE. Neonatal Hypoglycemia. Pediatrics. 1999;20(6)

3. Mnistry of Health $(\mathrm{MOH})$, Situation analysis of Newborn health in Uganda; Current status and opportunities to improve care and survival 2008.

4. World Health Organization (WHO),Neonatal hypoglycemia1997.

5. Special Care Unit, Fluid requirements and management in neonates. 2011.

6. Nili F, Ghafuri M. Hypoglycemia in sick preterm infants and the therapeutic effect of 12.5\% Dextrose in water compared with $10 \%$ Dextrose in water. Acta Medica Iranica. 2005;43(3).

7. PS power and sample size program available for free on the Internet. Controlled Clin Trials [database on the Internet]1997.

8. Lilien LD, Grajwera LA, Rosita SP. Treatment of neonatal hypoglycemia with continuous intravenous glucose infusion. Journal of Pediatrics. 1977;91(5):77982.

9. Alaroker F. Prevalence, clinical features and possible risk factors of neonatal hypoglycemia occuring within 24 hours of birth admiited to SCU, Mulago hospital. Kampala: Makerere; 1999.

10. Anil M. prevention and management of neonatal hypoglycemia. Arch Dis Child. 1994;70:F54-F65.

11. Lilien LD, Rosita P, Srinivasan G, Sambasivarao V, Tsu Y. Treatment of neonatal hypoglycemia with minibolus and intravenous glucose infusion. Journal of Pediatrics. 1980;97(2).

12. Namukwaya J. Survival of Very and extremely low birth weight Neonates admitted to Special care unit of Mulago Hospital; A prospective study. Kampala: Makerere college of Health Sciences; 2011.

13. Vander LM, Vivianne EHJ, Smits W, Paul HT, Van Z, Frans JW. Short term and long term outcome of neonatal hyperglycemia in very preterm infants: A retrospective follow up study. BMC Pedaitrics. 2010;10(52):1471-2431.

14. Louik C, Allen A, Mitchell, Epstein MF, Shapiro S. Risk factors for Neonatal hyperglycemia Associated with 10\% Dextrose Infusion. AJDC. 1985;139.

15. Hamid MH, Chishti AL, Magbool S. Clinical utility and accuracy of a blood glucose meter for the detection of neonatal hypoglycemia. Journal of the college of physicians and surgeona - Pakistan. 2004;14(2):225-8. 